\title{
Comparative Study of Partial Discharge Localisation Techniques within Transformer Windings
}

\author{
M. S. Abd Rahman \\ Universiti Tenaga Nasional \\ UNITEN-IKRAM, Putrajaya Campus \\ 43000, Selangor Darul Ehsan, Malaysia \\ asafwan@uniten.edu.my
}

\author{
P. L. Lewin* and P. Rapisarda \\ *The Tony Davies High Voltage Laboratory \\ University of Southampton \\ University Road, SO17 1BJ \\ Southampton, United Kingdom
}

\begin{abstract}
Partial discharge (PD) activity may occur inside power transformers and the existence of this activity produces a negative effect on the transformer until eventually there is catastrophic failure. Therefore, partial discharge detection and measurement within a transformer winding have become an important research area to provide asset health information enabling the maintenance and replacement processes to be carried out effectively. A modern condition monitoring system for PD activity inside HV transformers may use any observable phenomena but here we concentrate on an electrical detection method. The fundamental localization technique uses wavelet multi-resolution analysis for calculating the energy at different frequency ranges and Principal Component Analysis as dimensional reduction tool that produces the output that ultimately can be used as an indicator of PD location inside a transformer winding. However, an alternative method has been developed to using corresponding 3 dimensional filters to enable the localisation process. Both approaches are compared and obtained results are presented in this paper.
\end{abstract}

Keywords-Partial discharge; transformer; condition monitoring

\section{INTRODUCTION}

In high voltage transformers, partial discharge (PD) can occur due to ageing and degradation processes of the insulation systems. PD activities within transformers, which occur over time, can be due to several factors such as thermal and electrical overstressing. At early stages of operation, this activity could be due to defects introduced during the manufacturing process. Ultimately, these processes can increase the likelihood of further degradation of the insulation system and lead to a catastrophic failure if untreated. To avoid this, regular condition monitoring $(\mathrm{CM})$ is needed $[1,2]$ to rapidly identify the presence of a degradation process. There are several condition monitoring approaches for high voltage transformers, initially this was based on time preventative maintenance which were performed periodically regardless of asset condition. The disadvantages of this approach are higher operational costs and undetectable imminent failure. Therefore, $\mathrm{CM}$ practice is moving from a time-based approach to being response based on condition assessment strategies some of which are 'on-line'. However, on-line condition monitoring requires advanced detection, measurement and analysis systems in order to successfully perform any assessment. It can then reliably provide asset health information which enables maintenance and replacement processes to be carried out effectively. Therefore, condition monitoring for large assets such as high voltage transformers in terms of partial discharge have become an important research area in recent years and there have been intensive studies covering many important factors for effective condition monitoring of high voltage transformers $[2,3,4]$. Generally, different sensors have been applied to detect different forms of partial discharge energy such as electrical signals or acoustic or electromagnetic waves. This work uses earth current measurement as a PD measurement technique to detect any $\mathrm{PD}$ signals and radio frequency current transducers (RFCT) have been found to be an effective sensing method and have been proven to be sensitive enough for PD measurement of discharge currents over a frequency range of $10 \mathrm{kHz}$ to $200 \mathrm{MHz}$. In this case, the signals are detected flowing from discharge source location to measurement points, which are grounded via the bushing tap and neutral to earth connections [5]. Within the laboratory, real PD sources such as a void discharge, surface and corona discharges have been used to simulate PD activity inside an interleaved type winding to create PD data in order to develop and test proposed analytical methods. More recent research of PD analysis of large electrical assets has been reported [6]. Initial work at Southampton [7] used the Wavelet transform (WT) to decompose PD signal into different frequency and time domains. Meanwhile, data mining techniques such as Principal Component Analysis (PCA) and t-Distributed Stochastic Neighbour Embedding (t$\mathrm{SNE}$ ) to represent the distribution of energy of the captured PD pulse for PD source discrimination. Hence, a similar methodology has been applied to provide an indicator of PD location inside a transformer winding [8]. Ultimately, developing this method into real-time autonomous condition monitoring, this method has been implemented using finite impulse response filters (FIR) to directly perform this combined operation and it has significant potential to replace a standard off-line operation [9]. Moreover, the results that have been presented are very promising as an indicator of PD location within a transformer winding. Hence, this paper presents a comparative study between the fundamental 
approach and a real-time friendly approach using different PD sources. The experiment in order to simulate PD activities within a high voltage transformer winding is discussed in the next section in this paper and the fundamental method, as well as 3 dimensional designed filters method are discussed in the following section. Obtained results using both methods are presented and compared before a summary of the conclusions resulting from this work.

\section{EXPERIMENT}

The experiment consists of a high voltage transformer winding model BS148:1998 class 1, which was manufactured by Alstom and Stanlow Works, $60 \mathrm{kV}$ transformer bushing $60 \mathrm{HC} 755$, artificial partial discharge sources as well as a PD detection and measurement system. The whole transformer winding is immersed in oil, the transformer and measurement systems are PD free up to $30 \mathrm{kV}$. Figure 1 shows a schematic diagram of the experiment to inject signals into transformer winding using PD sources which are immersed in silicone oil due to its properties and also used in transformer. The transformer has two types of windings, an interleaved disc and a plain disc winding that remains grounded during experiments; in this case the PD signal was generated and injected into the interleaved disc winding section. The interleaved winding consists of eight external terminals which are internally interconnected to eight sections with two discs for every section, for replicating a real high voltage transformer, terminal one of the winding is connected to a bushing core bar while the last terminal was grounded. Artificial PD sources such as void discharge, corona discharge, surface discharge and floating discharge were used to generate impulse type signals which were then injected at each terminal of the winding via a cable. The current pulses from every section of the winding which initiated by the PD activities are measured at both allocated measurement points. The selection of measurement points is based on the assumption that signals from the PD sources are travelling in both directions towards an earth point, thus, there are two external measurement points located at the bushing tap to earth which is connected to terminal 1 while the other is at the neutral to earth connection and connected at terminal 8 .

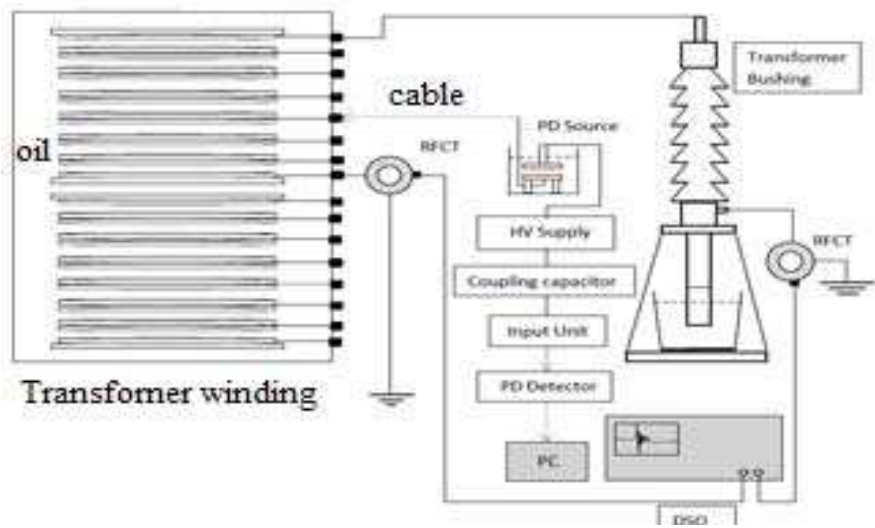

Figure 1 A schematic diagram illustrates experiment to simulate PD inside a transformer winding in laboratory.

\section{LOCALISATION METHODS}

a. Wavelet Transform and PCA

The wavelet transform is a useful mathematical tool for timefrequency domain analysis. It has been applied effectively of partial discharge analysis in high voltage plant [7]. The basis of the wavelet transform requires selection of a proper mother wavelet $(\psi(\mathrm{m}))$ according to the analysed signal. There are various types of mother wavelet, such as Symlet, Meyer, Coiflet, Morlet and Daubechies wavelet which have unique properties and are suitable for different applications. In this paper, the Daubechies wavelet is applied due to the fact that this mother wavelet was found in the initial investigation to be able to 'map' the characteristics of the PD pulse and hence is believed to be effective in analysis of the transient signal produced by PD activity [7].

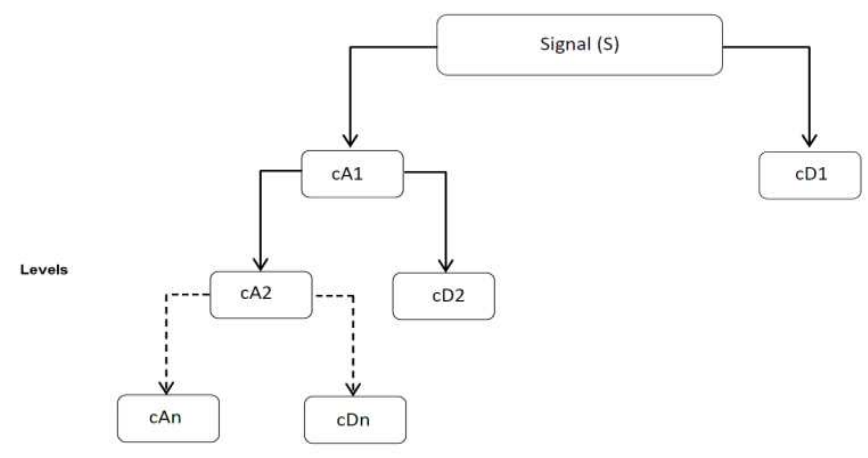

Figure 2 Iterative process of Wavelet Transform (WT)

Principal component analysis is a non-parametric statistical method that is used widely and is the most popular dimensional reduction technique for large data sets and can reveal hidden patterns inside data [1]. Wavelet analysis generates a distribution of $(n+1)$ energy variables over the frequency range. As such the individual PD pulse can be represented by a vector of these ten variables (or a single point in $(\mathrm{n}+1)$ dimensional spaces). In this paper, just three variables are used for visualization of the features hidden in the data sets, thus, PCA was used to extract these three values that best represent the $(\mathrm{n}+1)$ vector.

\section{b. 3 Dimensional FIR filters}

Ultimately, the 3 dimensional filters are derived based on system identification using a linear combination of Wavelet function and PCA weighting $[9,10]$. Hence, they can be approximated as Finite Impulse Response (FIR) filters. Each derived filter is unique and orthogonal to each other and is therefore similar to principal components. Due to the fact that fundamental results on this project have found that PD localisation within transformer winding can be determined using three principal components and also the use of visualisation is only possible within 3 dimensional thus only three FIR filters were constructed. Figure 3 shows 3 
dimensional filters that can be used for PD localisation within transformer windings. The detail of FIR filters construction and application on PD localisation can be found in [11].

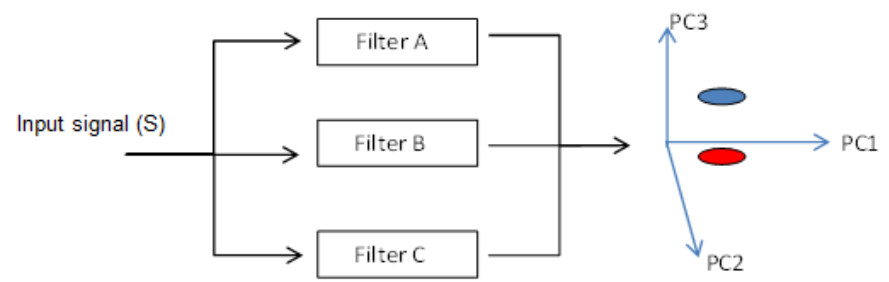

Figure 3 A schematic diagram of 3 dimensional filters for on-line application using wavelet and PCA which can be used as a PD localisation tool.

\section{RESULTS AND DISCUSSIONS}

Ideally, the investigation of PD location within a transformer winding is to use the energy distributions from the Wavelet Transform and PCA as a dimensional reduction technique to select the three highest variances that are uncorrelated within measurement data. Thus, the process will generate coordinate points as known as principal component scores which are representative of the original data. The plot data have been normalized to unity for comparison purposes.

\section{a. Wavelet Tranforms and PCA}

It is believed that the travelling signal propagating through a transformer winding, contains an energy distribution in time and frequency corresponding to the 'distance' of PD activity from the measurement sensors. Ultimately, the different energy will cause variations of cluster positions of groups of pulses detected at each measurement point. Figure 4 reinforces the hypothesis as it indicates that different PD source locations will produce different separations between two clusters i.e. the blue and the red clusters represent the bushing tap point and the neutral to earth connection measurements respectively.

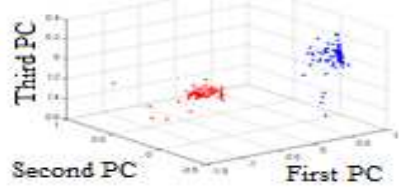

a.

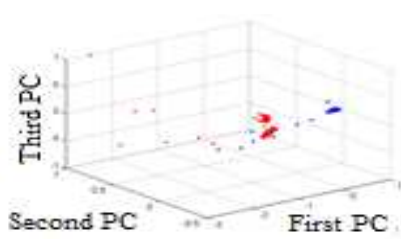

c.

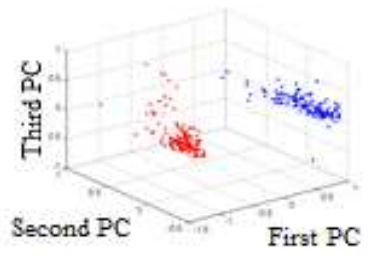

b.

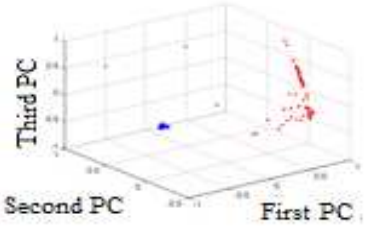

d.
Figure 4 A plot of cluster pattern for a combined Wavelet Transform and PCA approach a terminal $1, \mathrm{~b}$ terminal 3 , c terminal $6, \mathrm{~d}$ terminal 8

\section{b. Three dimensional FIR filters}

There are in total four PD sources that have been used in the investigation and from each PD source, 200-300 PD pulses have been recorded for further analysis. Combined analysis utilizing the Wavelet Transform and PCA indicates that the location of the PD sources within a winding can be determined (Figure 4). Hence, implementation of designed filters has been undertaken and the results obtained are shown in Figure 5. The blue and red clusters represent PD data from the bushing tap point and neutral to earth connection respectively. Referring to Fig 5a. where the PD source is injected at terminal 1 of transformer winding. The blue clusters appear at the upper side of the plot, thus it can be inferred that the energy of the clusters are higher than the red clusters. On the other hand, when the PD source is injected at terminal 8, which is interconnected with a connection to ground, the plot is reversed where the red clusters are at the upper part of the plot, showing that the energy of these pulses have greater energy than the bushing tap point measured pulses. Meanwhile, Figure 5c shows the cluster pattern when the PD source is injected at terminal 5 of the transformer winding.

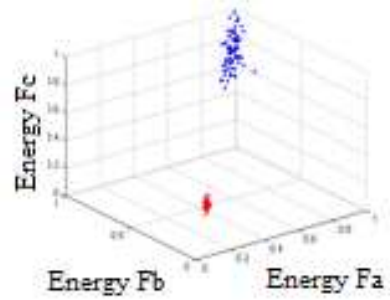

a.

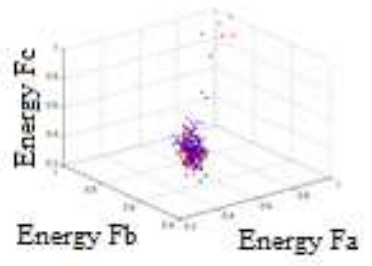

c.

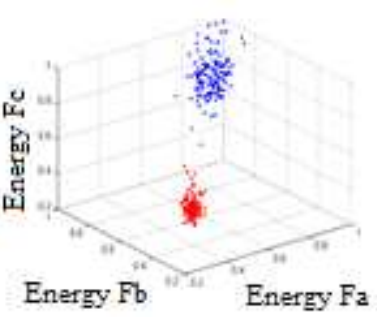

b.

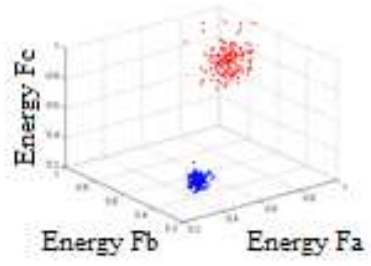

d.
Figure 5 A plot of cluster pattern for designed filters approach a terminal $1, \mathrm{~b}$ terminal 3, c terminal 5, d terminal 8

\section{c. Comparison of Wavelet PCA and 3D filters}

This comparison is based on PD measurement data and using two different approaches in order to determine PD location within a transformer winding where the first approach is a fundamental technique while the second may be applied for on-line condition monitoring of partial discharge. Therefore, the results obtained from both approaches are compared in order to provide an overview and ultimately a conclusion whether the second approach which developed based on fundamental approach can be used further in PD localisation. Due to the fact that these two approaches are using different variables i.e. principal component coordinates and energy 
respectively, the cluster plots cannot be compared. Nevertheless, the separation distance which is dimensionless can still be used regardless of the position of the blue and red clusters in the respective spaces, as the separation between them would correspond to the place where the PD activity has occurred.

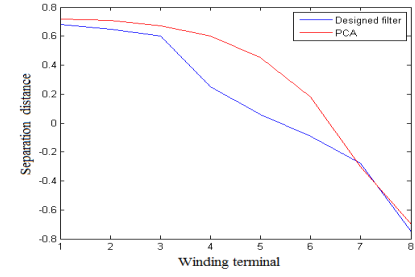

a.

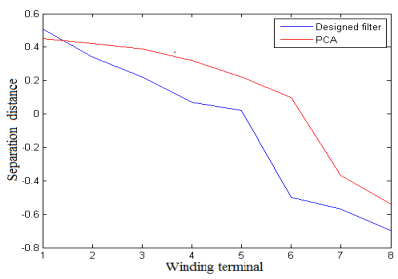

c.

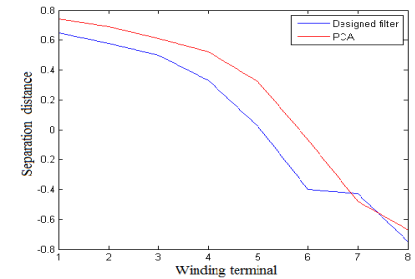

b.

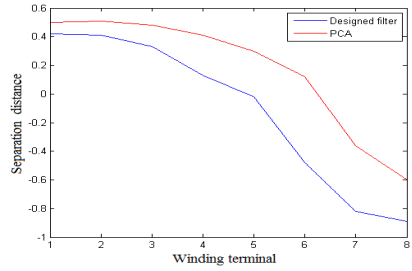

d.
Figure 6 Comparison of Wavelet PCA approach and the design filter approach a. void discharge $b$. surface discharge c. corona discharge d. floating discharge

\section{CONCLUSION}

The localisation process of partial discharge sources within a transformer winding is primarily based on measurement data obtained using standard radio frequency detection and sensors. The measurement data from the bushing and neutral to earth connection are in terms of electrical pulses which need to be analysed in order to extract useful information such as energy using the fundamental technique of applying the Wavelet Transform and PCA. Obtained results from the initial approach have shown the cluster patterns can be used to indicate PD location. Thus, this research has moved to a next step to enable the localisation process to be carried out without human intervention in real-time basis using standard FIR filters. The designed filters have been constructed based on Wavelet filters as well as PCA thus it is assumed that the results produced by both approaches are comparable. Therefore, the separation distance produced from both approaches are compared for different types of PD sources.
The similar pattern of separation distance of the blue and red clusters have been shown using two different approaches. However, there is an "offset" due to the different positive and negative spaces in the PCA plots, which are not in the energy plots produced by designed filters.

\section{ACKNOWLEDGMENT}

The first author would like to thank University of Southampton and The Tony Davies High Voltage Laboratory for funding this project. Also to Prof. Paul Lewin and the condition monitoring research team of TDHVL for sharing data.

\section{REFERENCES}

[1] M. S. Abd Rahman, P. Rapisarda and P. L. Lewin, "Partial discharge propagation inside a high voltage transformer winding," Int. Conf. Electrical insulation and dielectric phenomena, 2012, pp. 56-59.

[2] M. D. Judd, L. Yang and I. B. B. Hunter, "Partial discharge monitoring of power transformers using UHF sensors. Part 1: sensors and signal intepretation,'IEEE Electrical Insulation Magazine, 21(2), March-April 2005, pp. 5-14.

[3] T. Saha, "Review of modern diagnostic techniques for assessing insulation condition in aged transformers," IEEE Trans. on Dielectrics and Electrical Insulation, 10(5), 2003, pp. 903-917.

[4] P. Werle, H. Borsi and E. Gockenbach,"A new method for partial discharge location on power transformers based on a system theoretical approach", Int. Conf. on Prop. \& App. of Dielectric Materials, 2000, pp. 831-834.

[5] P. L. Lewin, I. O. Golosnoy and R. Mohamed, "Locating partial discharge sources in high voltage transformer windings," Electrical Insulation Conference, pp. 196-200, June 2011.

[6] P. L. Lewin, "On-line partial discharge analysis of transmission and distribution assets", IEEE Electrical Insulation Conf. Philadelphia, USA, June 2014, pp. 180-184.

[7] L. Hao and P. L. Lewin, "Partial discharge source discrimination using a support vector machine", IEEE Trans. Dielectr. Electr.Insul., Vol. 17, 2010, pp. 189-197.

[8] M. S. Abd Rahman, P. Rapisarda and P. L. Lewin, "The study of the effect in variation of A.C. applied voltage to the partial discharge localization inside transformer windings," Int. Conf. Electrical insulation and dielectric phenomena, 2013. Montreal, Canada,

[9] M. S. Abd Rahman, P. Rapisarda and P. L. Lewin, "Construction of finite impulse wavelet filter for partial discharge localisation inside a transformer winding," Int. Conf. Electrical Insulation, June 2013, Ottawa, Canada, pp. 30-34.

[10] Z. Vana and H. A. Preisig, "System identification in frequency domain using wavelets: Conceptual remarks", Sys. \& Cont. Letters, July, 2012.

[11] M. S. Abd Rahman, P. L. Lewin and P. Rapisarda, "The use of three dimensional filters for on-line partial discharge localisation in large transformers", IEEE Elec. Insulation Conference, Philadelphia, USA, 811 June 2014, pp. 10-14. 